\title{
FUNDAMENTOS DA NUTRIÇÃO NA PRÁTICA DE PILATES®: UMA REVISÃO DE LITERATURA.
}

\section{NUTRITION FUDAMENTS IN PILATES® PRACTICE: A LITERATURE REVIEW.}

\author{
Neidi Aparecida Lisboa DA LUZ1'; Caryna Eurich MAZUR²; Aline Jabur CASTILHO³
}

1 - Graduanda em Nutrição. Faculdade Campo Real de Guarapuava - PR, Brasil. E-mai

2 - Departamento de Nutrição. Universidade Estadual do Centro-Oeste, Paraná, Brasil.

3 - Colegiado de Nutrição. Faculdade Campo Real, Paraná, Brasil.

Autor para correspondência: neidyy15@gmail.com

\section{RESUMO:}

O objetivo desta pesquisa foi analisar a relação existente entre o comportamento alimentar e a atividade física, ressaltando os benefícios que o Método Pilates $₫$ proporciona aos seus adeptos na composição corporal e qualidade de vida. Este estudo constitui-se de uma revisão integrativa de literatura. As buscas foram realizadas nas bases de dados Bireme, LILACS, Scielo para selecionar artigos originais publicados entre 2001 a 2017. Por meio de uma análise descritiva, observa-se o aumento de casos de sobrepeso e obesidade nos dias atuais, estando relacionados aos hábitos alimentares errôneos e a falta de atividade física. O Método Pilates ${ }^{\circledR}$ surge então, como proposta de atividade física, nota-se que o peso corporal dos praticantes recebe influência devido o aumento do gasto energético induzido pelo esforço físico realizado. Uma alimentação saudável aliada à prática de atividade física são fatores determinantes para uma melhor qualidade de vida. O Método de Pilates $®$ além de proporcionar muitos benefícios, surge como uma proposta de atividade física, contribui na redução de peso e medidas, na mudança da composição corporal com o aumento de massa magra sem sobrecarregar o organismo. No entanto, seriam necessárias mais pesquisas na área para afirmar os resultados.

Palavras-chave: alimentação, antropometria, composição corporal, Pilates, atividade física.

\section{ABSTRACT:}

The objective of this research was to analyze the relation between eating behavior and physical activity, highlighting the benefits that Pilates $\AA$ Method provides to its adepts in body composition and quality of life. This study is based on an integrative literature review. The searches were made on the Bireme, LILACS and Scielo databases to select original articles published between 2001 and 2017. Through a descriptive analysis, it is observed the increase in overweight and obesity cases nowadays, being related to erroneous eating habits and lack of physical activity. The Pilates ${ }^{\circledR}$ Method comes up then, as a proposal of physical activity, and it is noted that the body weight of the practitioners receives influence due to the increase of energy output caused by the physical effort performed. Healthy eating habits allied to the practice of physical activity are determinant factors to a better quality of life. The Pilates $₫$ Method besides providing several benefits contributes to reduce weight and measures, change of body composition with increase of lean mass without overwhelming the organism. However, it would be necessary more research in the field to affirm the results.

Key words: nutrition, anthropometry, body composition, Pilates, physical activity. 


\section{INTRODUÇÃO}

A atividade física é reconhecida como fator de proteção para a saúde, seus benefícios são inúmeros e estão associados à redução de risco para doenças crônicas como a diminuição do risco de morte precoce por doenças cardiovasculares na manutenção do peso corporal (SILVA et al., 2017). No entanto, a falta de atividade física causa uma desordem biológica, o que contribuiu para o crescimento da prevalência de sobrepeso e obesidade mundialmente (SOUSA E VIRTUOSO JUNIOR, 2005).

Segundo Motta Junior (2014) diferentes componentes corporais sofrem alterações durante a vida toda, o que torna a composição corporal uma característica dinâmica, influenciada por aspectos fisiológicos, como crescimento e desenvolvimento além do aspecto nutricional.

Sabe-se que as mulheres têm maior deposição de gordura corporal. Ainda, além dos maus hábitos alimentares, a inatividade física, os aspectos fisiológicos a obesidade também pode estar ligada a alterações endócrinas e emocionais (OLIVEIRA E SILVA, 2014).

De acordo com as informações obtidas pela Vigilância de Doenças Crônicas por Inquérito Telefônico (VIGITEL, 2016), a obesidade cresceu 60\% em dez anos, de 11,8\% em 2006 para 18,9\% em 2016.

O aumento pode ter como causa principal o consumo excessivo de dietas com alta densidade energética, alimentos ricos em gorduras saturadas e açúcares além da atividade física reduzida (SOUSA E VIRTUOSO JUNIOR, 2005; VIGITEL, 2016).

O nível regular de atividade física acompanha benefícios, os quais se manifestam sob todos os aspectos do organismo, seja ele musculoesquelético, melhora da força e do tônus muscular, da flexibilidade, fortalecimento dos ossos e das articulações, redução de peso e medidas (PICOLLI, 2010). Especialistas creditam várias atividades físicas que podem fortalecer o corpo humano, como por exemplo: o Pilates. Consagradamente, Joseph Hubertus Pilates desenvolveu o Método Pilates $\AA$ durante a $1^{\text {a }}$ Guerra Mundial. O programa de exercícios idealizado por Joseph obedece a seis princípios: concentração, controle, centralização de força, fluidez, precisão e respiração (DA COSTA; ROTH E NORONHA, 2012).

O Método Pilates ${ }^{\circledR}$ é simples e não apresenta riscos à saúde dos praticantes, possui poucas contraindicações, e pode ser indicado até mesmo para aqueles que são proibidos de participar de determinados programas de exercícios convencionais. Os 
exercícios são prescritos conforme as necessidades e de acordo com os objetivos a serem alcançados, pois a frequência da atividade deve respeitar os níveis de aptidão e habilidade física individual (PICOLLI, 2010).

Estudos mostram que os benefícios e aplicações do Método Pilates® são inúmeros, também direcionados a reabilitação e promoção da saúde de diferentes populações (PICOLLI, 2010; DA COSTA; ROTH E NORONHA, 2012; MARTINS, 2013, COUTINHO, 2017).

O peso corporal dos praticantes de Pilates ${ }^{\circledR}$ pode receber influência, devido ao aumento do gasto energético induzido pelo esforço físico realizado, se tornando uma alternativa para quem procura a perda de peso. Os valores do peso corporal dependem de alguns componentes como o peso da gordura corporal e o peso de massa magra (músculos, ossos, água) que, dependendo da alimentação habitual e o tipo de atividade física praticada sofrem diferentes variações (SIMAS; KESSLER E SANTOS, 2010; NOGUEIRA et al., 2014).

Dessa maneira, este estudo objetivou revisar a relação existente entre o comportamento alimentar e a atividade física, ressaltando os benefícios que o Método Pilates $®$ proporciona aos seus adeptos em sua composição corporal e qualidade de vida.

\section{MÉTODOS}

Trata-se de uma revisão simples de literatura. O método de pesquisa permitiu a busca, avaliação crítica e a síntese das evidências disponíveis do tema investigado.

Foram percorridos diversos passos para a construção dessa revisão: (1) definição da questão norteadora: analisar a relação existente entre o comportamento alimentar e a atividade física, ressaltando os benefícios que o Método Pilates® pode proporcionar aos praticantes; (2) estabelecimento de critérios para a seleção de artigos; (3) leitura dos artigos; (4) análise crítica das informações; (5) interpretação e apresentação dos resultados obtidos

Para a seleção dos artigos foi utilizado o acesso online a três bases de dados: Bireme(Centro Latino-Americano e do Caribe de Informação em Ciências da Saúde), LILACS (Literatura Latino-Americano de Ciência e Saúde) e Scielo (ScientificElectronic Library Online). As buscas nas três bases ampliaram o âmbito de pesquisa.

A procura dos trabalhos foi realizada entre os meses de julho de 2016 a novembro de 2017. Os artigos e dissertações foram selecionados por meio de Descritores em 
Ciências da Saúde (DeCS), EBSC (Information Services is Partnering with Biblio Labs to Support Open Access Disconvery of Eletronic The sesand Dissertations): "alimentação", "antropometria", "composição corporal", "Pilates ${ }^{\circledR}$ ", “atividade física” na língua portuguesa.

Os critérios de inclusão definidos para a presente revisão foram: artigos, dissertações e periódicos completos disponíveis eletronicamente em idioma português, entre 2001 e 2017.Foram utilizadas pesquisas originais e algumas revisões sistemáticas, com abordagem qualitativa, quantitativa e descritiva, com variáveis de diagnóstico nutricional, composição corporal em mulheres, hábitos alimentares, comportamento alimentar e benefícios do Método Pilates ${ }^{\circledR}$.Os demais artigos, dissertações e periódicos que o título e o resumo não apesentavam relevância a pesquisa.

Foram incluídos, à princípio, cinquenta e um artigos, doze dissertações e sete periódicos, dentre os revisados vinte e seis foram utilizados. Dos vinte e seis revisados, nove artigos, duas dissertações e dois periódicos referiam-se diretamente ao Método de Pilates ${ }^{\circledR}$ e composição corporal. Os demais, sendo dez artigos, uma dissertação e dois periódicos sobre comportamento alimentar, obesidade e atividade física em mulheres nos dias atuais. Por meio da revisão integrativa de literatura foi possível obter informações pertinentes sobre a relação entre o comportamento alimentar, composição corporal e os benefícios proporcionados pelo Método de Pilates ${ }^{\circledR}$. Os resultados foram avaliados descritivamente.

\section{RESULTADOS E DISCUSSÃO}

\subsection{Princípios da alimentação}

Os hábitos alimentares são adquiridos de maneira gradual durante a primeira infância, e permanecem durante a vida adulta. No entanto, estes podem sofrer modificações, devido às mudanças na rotina e no estilo de vida (ROSSI; MOREIRA; RAUEN, 2008).

De acordo com os princípios de uma alimentação saudável, a alimentação diária deve ser composta por todos os grupos alimentares. A ingestão hídrica é essencial, assim como, os carboidratos, proteínas, lipídios, vitaminas, fibras e minerais, os quais são indispensáveis para o bom funcionamento do organismo. A alimentação deve ser adequada às diferentes fases e condições de vida. Deve conter alimentos de qualidade, os quais satisfaçam todas as necessidades do corpo, devem ser nutritivos e não apenas conter 
calorias vazias. Ser suficiente, equilibrada e variada para atender o organismo em todas as suas necessidades (ROSSI; MOREIRA E RAUEN, 2008).

Para Amoras et al. (2007) os hábitos alimentares, aversões e preferências estão intimamente ligados às identidades étnicas e culturais, são determinadas pelos costumes, crenças e alimentos disponíveis. A identificação e conhecimento de distorções alimentares incompatíveis com a preservação da qualidade de vida constituem-se hoje em dados importantes para a avaliação clínica de indivíduos, que apresentam risco para desenvolvimento de obesidade.

Os novos hábitos alimentares foram adotados devido às indústrias alimentícias e a praticidade e disponibilidade que elas oferecem ao consumidor. Observa-se uma redução na ingestão de produtos tradicionais (AMORAS et al., 2007).

Santos et al. (2012) afirmam as necessidades nutricionais como a ingestão de nutrientes que promovem e restabelecem o equilíbrio fisiológico. Assim, os hábitos alimentares resultam da resposta de um indivíduo ou de grupos às cominações sociais e culturais quanto à disponibilidade, escolha e utilização de reservas alimentares.

A alimentação saudável e adequada é um fator determinante e condicionante da saúde, é um direito de todas as pessoas. A alimentação e nutrição estruturam-se no contexto da segurança alimentar e nutricional, integrando um conjunto de ações que visam respeitar, proteger, promover e favorecer o direito humano à saúde e à alimentação (ALVES E JAIME, 2014).

\subsection{Alimentação na atividade física}

A prática regular de atividade física é essencial em qualquer idade, considera-se um meio de preservar e melhorar a saúde e a qualidade de vida do ser humano. A inatividade física aumenta significativamente o risco de doenças crônicas (FREIRE et al., 2014).

Segundo Rogatto e Gobbi (2001) e Coutinho (2007) os novos hábitos alimentares e a falta de atividade física causam uma desordem biológica, diversos sistemas são afetados negativamente, o que contribui para o crescimento da prevalência de sobrepeso e obesidade. No momento atual, a obesidade é considerada um dos maiores problemas de saúde pública, as causas e complexidade são um desafio para muitos especialistas da área da saúde.

Segundo informações do Instituto Nacional de Alimentação e Nutrição (2005), 32\% 
da população brasileira apresenta algum tipo de alteração na composição corporal. A proporção de sobrepeso e obesidade aumenta com a idade, sendo 37\% nos homens e 55\% nas mulheres. O crescimento pode ter como causa principal o consumo excessivo de dietas com alta densidade energética, ricos em gorduras saturadas e açúcares além da atividade física reduzida (SANTOS et al., 2012).

Pesquisas realizadas pela Vigilância de Doenças Crônicas por Inquérito Telefônico (VIGITEL, 2016) retratam o sobrepeso e a obesidade como uma epidemia com a incidência e prognóstico insatisfatório para a saúde pública. O problema é mais comum entre os homens, passou de 47,5\% em 2006 para 57,7\% em 2016. No entanto, houve um aumento significativo entre as mulheres, o índice passou 38,5\% para 50,5\%, tornando-se ainda maior com o avanço da idade em ambos os sexos. A pesquisa ressalta a influência dos hábitos alimentares, a falta de atividade física e o grau de escolaridade.

Sabe-se que o comportamento alimentar, ou seja, os hábitos alimentares do ser humano, é um dos fatores condicionantes para o seu estado nutricional (LINO; MUNIZ; SIQUEIRA, 2011; VIGITEL, 2016).

Inúmeros autores afirmam que hábitos alimentares errôneos se constituem como fator decisivo para o surgimento de sobrepeso e posteriormente o desenvolvimento da obesidade (ROGATTO E GOBBI, 2001; COUTINHO, 2007; LINO; MUNIZ; SIQUEIRA, 2011; SANTOS et al., 2012).

É essencial entendermos que os termos sobrepeso e obesidade frequentemente utilizados, não possuem o mesmo significado. Sobrepeso refere-se ao aumento do peso corporal total, o qual excede o peso normal de um indivíduo. Obesidade caracteriza-se pelo aumento generalizado ou localizado de tecido adiposo em relação ao peso corporal, e ainda pode estar associada a riscos para a saúde (LIMA et al., 2011).

Segundo Miranda e Morais (2002), a composição corporal se refere a quantidades relativas de distintos compostos corporais, os quais possibilitam uma estimativa de massa muscular e massa gorda, sendo relevante na determinação, monitoramento da saúde e no condicionamento físico, além de facilitar o planejamento de programas de treinamento para atletas e praticantes de atividade física. Alimentação balanceada aliada a atividade física são fatores decisivos para uma boa saúde, controle de gordura corporal e melhor qualidade de vida.

Diversos estudos constataram que um dos fatores que contribui para uma melhor qualidade de vida é a prática regular de atividade física. No entanto, esta deve ser adequada e orientada adequadamente conforme a necessidade de cada indivíduo (MIRANDA E MORAIS, 2002; SIMAS; KESSLER E SANTOS, 2010). 


\subsection{Pilates ${ }^{\circledR}$}

De acordo com Perfeito et al. (2016), a pratica do Método Pilates® apresenta-se como um aliado na promoção da saúde, indica possibilidade de ganhos nos padrões de resistência, força e flexibilidade.

Para Macedo, Haas e Goellner (2015), esse método vem se destacando por proporcionar melhorias acentuadas em diversos aspectos neuromusculares. Os benefícios do método vão além do ganho de força e resistência muscular, promove a interação corpo e mente, melhora na capacidade respiratória, aumento da flexibilidade, fortalecimento, restruturação do corpo, prevenção de lesões, aumento no reconhecimento e identificação dos processos e movimentos corporais. Inclui também melhora na autoestima e diminuição de dores musculares.

Segundo estudos os benefícios e aplicações do Método Pilates® são inúmeros, também se direcionam a reabilitação e promoção à saúde de diferentes populações. Dependem unicamente da execução dos exercícios com fidelidade aos seus princípios voltados ao fortalecimento muscular. O Método Pilates ${ }^{\circledR}$ é a junção da abordagem oriental com a ocidental. As técnicas orientais visam o relaxamento, concentração, respiração, controle e flexibilidade, a ocidental dá ênfase ao movimento com força. Originando assim, a essência dos princípios do Método Pilates® (PERFEITOet al., 2016).

Segundo Miranda e Morais (2002), o interessante é que a atividade seja desenvolvida conforme as necessidades específicas de cada praticante. Os exercícios são planejados e adaptados de acordo as condições físicas e necessidades do praticante, não apresentando contraindicações.

O Método Pilates $\AA$ também contribui para a redução de peso e medidas, devido ao gasto calórico que proporciona durante a atividade, isto ocorre em conjunto com o ganho de massa muscular (COUTINHO, 2017).

Portanto é um exercício físico planejado para o condicionamento do corpo e da mente, corrige desequilíbrios musculares, melhorar a postura e tonifica a musculatura (NOGUEIRAet al., 2014).

\subsection{Nutrição e estado nutricional na prática do Pilates ${ }^{\circledR}$}

Sabe-se que o estado nutricional se refere ao equilíbrio entre a ingestão e a necessidade de nutrientes. As causas do excesso de gordura corporal são inúmeras. No 
entanto, o fator principal é o desequilíbrio energético, ou seja, o consumo calórico é maior que o gasto energético diário (MONDENEZE, 2007).

De acordo com Silva (2013), não basta diminuir a ingestão calórica para reduzir gordura corporal, uma vez que o organismo elimina do total do peso reduzido, apenas $30 \%$ de gordura e $70 \%$ de líquidos. Parte destes líquidos é originário da massa muscular, por essa razão, um dos resultados é a desagradável flacidez.

Em relação ao balanço energético, o oposto é verídico, não basta somente a prática de atividade física, é essencial a educação alimentar e nutricional. Visto que, se a ingestão de alimentos hipercalóricos excederem os níveis de energia gastos no dia, irá ocorrer o acúmulo de gorduras corporais (SILVA, 2013).

Segundo Coutinho, (2017) o Método Pilates® pode ser trabalhado de forma contínua com baixa intensidade, por meio de exercícios de solo, abaixo do limiar anaeróbio, utilizando a gordura como fonte de energia, a chamada lipólise. Ou por meio de exercícios mais intensos e intervalados, pode ser realizado nos equipamentos e com o uso de cargas, o que leva a aumentar a massa muscular, acelerando o metabolismo e isso contribui para o emagrecimento.

\section{CONCLUSÃO}

O método Pilates ${ }^{\circledR}$ além de proporcionar muitos benefícios, surge como uma proposta de atividade física, contribui na redução de peso e medidas, e na mudança da composição corporal devido ao gasto calórico proporcionado durante a atividade, isto ocorre em conjunto com o ganho de massa muscular sem sobrecargas ao organismo. Assim, constatou-se que o método se mostra eficaz em relação à composição corporal.

\section{REFERÊNCIAS}

ALVES, K. P. S.; JAIME, P. C. The national food and nutrition policy and its dialogue with the national food and nutrition security policy. Ciência \& Saúde coletiva, vol. 19, n. 11, p. 4331-4340, 2014.

AMORAS, R. D.; SOUZA, L.M.; SOUZA, N.M.F.; RASO, V. Relação entre hábitos alimentares e a composição corporal de funcionários da farmácia popular. Revista brasileira de obesidade, Nutrição e Emagrecimento, vol. 1, n.2, p. 72-77, Marc/Abr, 2007. 
CERRI A. S. Alimentação Saudável, Atividade Física e Qualidade de Vida. Roberto Vilarta (organizador). Campinas, IPES, Editorial, p. 90 - 94, 2007.

COUTINHO, W.; DUALI, P. Etiologia da obesidade. Revista da ABESO, v. 30, n. 30, 2007.

DA COSTA, L. M. R.; ROTH, A.; DE NORONHA, M. O método Pilates $\AA^{\circledR}$ no Brasil: uma revisão de literatura. ACM Arquivos Catarinenses de Medicina, vol.41, n.4, out.-dez. 2012.

DYNIEWICZ, A.M. Metodologia da Pesquisa em Saúde para Iniciantes. 3 ed. São Caetano do Sul, SP: Difusora Editora, 2014.

FREIRE, R.S.; LÉLIS, F.L.O; FONSECA FILHO, J.A. et al. Prática regular de atividade física: estudo de base populacional no Norte de Minas Gerais, Brasil. Revista Brasileira de Medicina do Esporte, vol. 20, n. 5, p. 345-349, 2014.

HISTÓRIA - AB Pilates ${ }^{\circledR}$ - Associação Brasileira de Pilates ${ }^{\circledR}$. Disponível em: < http:// www.abPilates ${ }^{\circledR}$.com.br/site/template.asp?idPagina=39> Acesso em:27/04/17.

LIMA, K. A.; SILVA, R.M.; SANTOS R.M.; et al. Efeitos da prática dos métodos Pilates®e musculação sobre a aptidão física e composição corporal em mulheres. Biológicas \& Saúde, v. 1, n. 1, 2011.

LINO, M. Z. R.; MUNIZ, P. T.; SIQUEIRA, K. S. Prevalência e fatores associados ao excesso de peso em adultos: inquérito populacional em Rio Branco, Acre, Brasil, 2007-2008 Prevalence of overweight and associated factors in adults: a population survey in Rio Branco. Cadernos de Saúde Pública, vol. 27, p. 797-810, 2011.

MACEDO, C. G.; HAAS, A. N.; GOELLNER, S. V. O método Pilates $\AA^{\circledR}$ no brasil segundo a narrativa de algumas de suas instrutoras pioneiras. Pensar a Prática, v. 18, n. 3, 2015.

MARTINS, R.A.S. Método Pilates ${ }^{\circledR}:$ Histórico, Benefícios e Aplicações Revisão Sistemática de Literatura. 2013. (Especialização em Pilates ${ }^{\circledR}$ do Centro de Estudos Avançados e Formação Integrada) - Universidade Católica de Goiás, 2013. 
MIRANDA, L.B.; MORAIS, P.D.C. Efeitos do Método Pilates® sobre a Composição Corporal e Flexibilidade. Revista Brasileira de Prescrição e Fisiologia do Exercício, vol.3, n.13, p.16-21. Jan/Fev. 2009.

MOTTA, J.J.B. Análise do Treinamento Resistido para boa Composição Corporal em Mulheres Jovens. 2014. 31 f. Dissertação de Graduação em Educação Física. Universidade Estadual da Paraíba, Centro de Ciências Biológicas e da Saúde. 2014.

NOGUEIRA, T.R.B.; OLIVEIRA, G.L.; OLIVEIRA, T.A.P. et al. Efeitos do Método Pilates® nas Adaptações Neuromuscular e na Composição Corporal de Adultos Jovens. Revista Brasileira de Prescrição e Fisiologia do Exercício. São Paulo. vol.8, n.45, p.296-303. Maio/Jun. 2014.

OLIVEIRA, A. P. S. V; SILVA, M. M. Fatores que dificultam a perda de peso em mulheres obesas de graus I e II. Revista Psicologia e Saúde, vol. 6, n. 1, p. 74-82, 2014.

Os 12 maiores benefícios do método Pilates ${ }^{\circledR}$ - Fisioterapia. Disponível em: < http://www.fisioterapia.comArtigo> Acesso em: 14/11/17.

PERFEITO, R.S.; MONTENEGRO, L.P.; GURGEL, A.V.; et al. Influência do tempo de intervalo entre séries e exercícios sobre o número de repetições e volume de um programa de atividades do método Pilates® Revista Carioca de Educação Física, vol. 11, p. 11-19, 2017.

PICOLLI, F. Efeitos do treinamento proporcionado pelo Método Pilates ${ }^{\circledR}$ Clássico nas Aptidões Físicas em mulheres saudáveis: um Ensaio Clínico Controlado. Universidade Federal do Rio Grande do Sul. Faculdade de Medicina. Programa de PósGraduação em Medicina: Ciências Médicas [Dissertação de Mestrado]. 2010.

ROGATTO, G. P.; GOBBI, S. Efeitos da atividade física regular sobre parâmetros antropométricos e funcionais de mulheres jovens e idosas. Revista Brasileira de Cineantropometria e desempenho humano, vol. 3, n. 1, p. 63-9, 2001.

ROSSI, A.; MOREIRA, E. A. M.; RAUEN, M. S. Determinants of eating behavior: a review focusing on the family. Revista de Nutrição, vol. 21, n. 6, p. 739-748, 2008. 
SANTOS, V.E.P.; CRUZ, N.M.; MOURA, L.A.; et al. O estado nutricional e comportamento alimentar de um grupo de mulheres na estratégia de saúde da família. Revisão de Literatura. Revista de Enfermagem da UFSM, vol. 2, n. 2, p. 394-400, 2012.

SILVA, A. S. O terceiro pilar do emagrecimento. In: Encontro Paranaense, Congresso Brasileiro de Psicoterapia Corporais, XVIII, XIII, 2013. Anais. Curitiba: Centro Reichiano, 2013. [ISBN - 978-85-87691-23-1] Disponível em: www.centroreichiano.com.br/artigos. Acesso em 07/10/2017.

SIMAS, A. R.; KESLLER, C.C.; SANTOS, P.P. Percepção da Qualidade de Vida de Adultos Praticantes do Método Pilates ${ }^{\circledR}$ em Estúdio em Florianópolis/SC. Revisão de Descritiva. Revista Brasileira de Prescrição e Fisiologia do Exercício, vol.4, n.22, p.363-369, Jul./Agost.2010

SOUSA, L.M.; VIRTUOSO Jr. J.S. A Efetividade de Programas de Exercícios Físico no Controle do Peso Corporal. Revisão de Literatura. Revista Saúde.Com., vol.1,p 71-78, Bahia, 2005.

VAZ, R.A.; LIBERALI, R.; CRUZ, T.M.F. et al. Método Pilates ${ }^{\circledR}$ na Melhora da Flexibilidade. Revisão Sistemática. Revista Brasileira de Prescrição e Fisiologia do Exercício, vol.6, n.31, p.25-31, Jan./Fev. 2012.

VIGITEL Brasil. 2016 - Ministério da Saúde. Disponível em:< http://www.portalarquivos.saude.gov.br/images/pdf/2017/junho/07/vigitel_2016_jun17.pdf. > Acesso em: 14/11/2017. 